\title{
Controle LFR Discreto de Quadrirotores usando o Framework ROS *
}

\author{
Lucas Schulze* Filipe K. Ijuim* Tiago J. M. Dezuo* \\ * Departamento de Engenharia Elétrica, Universidade do Estado de \\ SantaCatarina, Joinville/SC, (e-mails: schulze.lucass@gmail.com, \\ filk.ijuim@gmail.com,tiago.dezuo@udesc.br).
}

\begin{abstract}
This paper presents the development of the discrete version of the Linear Fractional Representation (LFR) control method for nonlinear systems, based on Linear Matrix Inequalities (LMIs). The main objective of the technique is to guarantee the stability of critical nonlinearities of the discrete-time system. Because of their inherent nonlinear and discrete-time properties, quadrirotors are used to illustrate the application of the method. As the LFR naturally usually has very aggressive control gains, a byproduct of the paper is the extension to avoid saturation of the control signal. An additional objective is the validation of the model/control in the ROS/Gazebo framework, which has internal models of commercial quadrirotors. This tool is based on realistic numerical simulations of systems based on their physical construction and forms, and is an excellent way of validating the dynamics equations used for the control design.

Resumo: Este artigo apresenta o desenvolvimento da versão discreta do método Linear Fractional Representation (LFR) de controle de sistemas não lineares, baseado em LMIs. O objetivo principal da técnica é a garantia de estabilidade de não linearidades críticas do sistema em tempo discreto. Devido as suas inerentes características não lineares e de tempodiscreto, os quadrirotores são utilizados para ilustrar a aplicação da técnica. Como o controle LFR naturalmente costuma apresentar ganhos de controle muito agressivos, um sub-produto apresentado é a extensão para que o sinal de controle não sature. Um objetivo adicional é a validação do modelo/controlador no framework ROS/Gazebo, que conta com modelos internos prontos de quadrirotores comerciais. Esta ferramenta tem como base simulações numéricas realistas de sistemas dinâmicos baseados em sua construção física e formas, sendo uma excelente maneira de validação das equações de dinâmica usadas para o projeto dos controladores.
\end{abstract}

Keywords: Nonlinear control; LFR; Discrete-time systems; quadrotors; ROS/Gazebo.

Palavras-chaves: Controle não linear; LFR; Sistemas em tempo discreto; quadrirotores; ROS/Gazebo.

\section{INTRODUÇÃO}

A crescente utilização em aplicações civis ocasionou em regulamentações cada vez mais rígidas para veículos aéreos não tripulados (VANTs). Para atender a essas normas, as técnicas de controle automático empregadas devem ser precisas e confiáveis. Entretanto, as características dinâmicas dos VANTs são altamente não lineares e com incertezas paramétricas, tornando necessária a utilização de técnicas de controle que apresentem garantias de robustez e o menor número de aproximações possível. Algumas dessas não linearidades, podem ser evitadas operando-se o sistema em uma região limitada, entretanto, outras não linearidades aparecem de forma inevitável e indissociável do comportamento do sistema, para as quais linearizações podem resultar em uma má representação do sistema real.

Um dos tipos mais comuns, os quadrirotores são VANTs de asas rotativas que apresentam quatro motores. No eixo de cada motor é fixada uma hélice que, ao rotacionar, gera

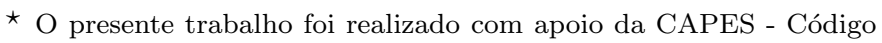
de Financiamento 001, e da FAPESC através do Edital No 04/2018. uma força de propulsão e um torque de rotação, responsáveis pela movimentação do quadrirotor. Normalmente os motores utilizados são do tipo Brushless DC (BLDC), que são acionados por um Electronic Speed Control (ESC), que por sua vez é comandado por um microcontrolador. Neste microcontrolador, embarcado no quadricóptero, é implementada a técnica de controle projetada para garantir a estabilidade deste sistema. Devido ao uso de microcontroladores digitais, necessita-se que as técnicas de controle sejam projetadas considerando tempo discreto. Portanto, é desejável a implementação de técnicas de controle de sistemas não lineares de tempo discreto.

Dentre as técnicas de controle não lineares com versão discreta disponível na literatura, pode-se citar as seguintes. A técnica conhecida como feedback linearization tem como característica principal o projeto de uma realimentação de estados não linear que cancele as não linearidades do sistema, permitindo um projeto de controle linear para o sistema de malha fechada (Grüne, 1997). Esta técnica tem como desvantagens restrições do sistema para que a realimentação seja um problema bem posto e o sistema deve ser bem conhecido para que o cancelamento seja 
efetivo. Outro método é o backstepping, cuja ideia é dividir um problema de projeto em uma sequência de subproblemas de ordem inferior, e recursivamente usar alguns estados como "controles virtuais" para obter as leis de controle intermediárias dos sub-problemas intermediários. Entretanto, sua classe de aplicações é limitada, apresenta sensibilidade numérica e dificuldades de ajustes de parâmetros (Pozo et al., 2008). Existem outras técnicas, como as baseadas em modos deslizantes e inteligência artificial, mas também com desafios no quesito robustez.

Uma técnica de controle de sistemas não lineares de interesse é a que utiliza Representação por Frações Lineares (LFR), que tem como base a solução de Desigualdades Matriciais Lineares (LMIs). Além de solução numérica eficiente, as LMIs também possuem como principais características a robustez na descrição de problemas com incertezas e a habilidade para considerar múltiplos requisitos de controle apenas anexando LMIs adicionais. Entretanto, não é conhecimento dos autores uma versão em tempo discreto do projeto de controle LFR.

Este artigo apresenta o desenvolvimento da versão discreta do método LFR de controle de sistemas não lineares, baseado em LMIs, motivado pela notada escassez da literatura. $\mathrm{O}$ artigo apresenta em detalhes a dedução da estratégia de controle para tornar acessível o desenvolvimento de extensões e adaptações do método, também omitidas da maioria das publicações relacionadas a LFR. O objetivo principal da técnica é a garantia de estabilidade de não linearidades críticas do sistema em tempo discreto. Devido a suas inerentes características não lineares e de tempodiscreto, os quadrirotores são utilizados para ilustrar a aplicação da técnica. Como o controle LFR naturalmente resulta em uma compensação do efeito das não linearidades, este costuma apresentar ganhos de controle muito agressivos. Para sistemas com limitações em seu sinal de controle, um sub-produto apresentado é a extensão para que o controle projetado não sature estes sinais.

Finalmente, um objetivo adicional com relação ao sistema é a validação da aplicação do controlador no framework ROS/Gazebo, que conta com modelos internos prontos de quadrirotores comerciais. Esta ferramenta, típica em simulações de robótica, tem como base simulações numéricas realistas de sistemas dinâmicos baseados em sua construção física e formas, sendo uma excelente maneira de validação das equações de dinâmica usadas para o projeto dos controladores.

Notação. $\mathbb{R}^{n}$ denota o espaço Euclidiano $n$-dimensional. $\mathbb{R}^{n \times m}$ é o conjunto de matrizes $n \times m$ reais. $\|\cdot\|$ simboliza a norma euclidiana de vetores e a norma espectral induzida de matrizes. $|\cdot|$ representa o valor absoluto de um número real. Blocos de matrizes que podem ser deduzidos por simetria são representados por $\star$. I é uma matriz identidade de dimensões adequadas. 0 é uma matriz de zeros de dimensões adequadas. Para uma matriz real $M, M^{\prime}$ denota sua transposta, $M>0(M<0)$ significa que $M$ é simétrica e positiva definida (negativa definida). c $(\cdot)$ e $\mathbf{s}(\cdot)$ representam, respectivamente, o cosseno e seno do ângulo em $(\cdot) \cdot \operatorname{diag}(\cdot)$ representa uma matriz diagonal formada pela sequência de elementos em $(\cdot) \cdot \bar{u}$ representa o valor máximo de $u$.

\section{MODELAGEM}

Neste capitulo é apresentada a modelagem do quadricóptero na configuração cruzada, em que os eixos $X$ e $Y$ do frame do corpo estão rotacionados $45^{\circ}$ em relação aos braços do drone, como mostra a Figura 1(b)-(c). A rotação $\phi$ em torno do eixo $X$ é chamada de roll, a rotação $\theta$ no eixo $Y$ de pitch, e a rotação $\psi$ em $z$ de yaw. Para realização de forças angulares e de empuxo, a velocidade de cada rotor deve ser modificada de formas diferentes, como pode ser visto na Figura 1.

(a)

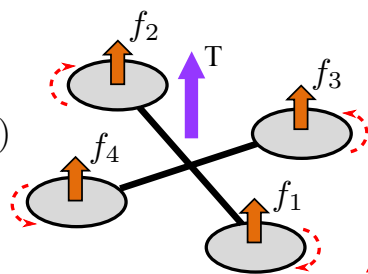

(c)
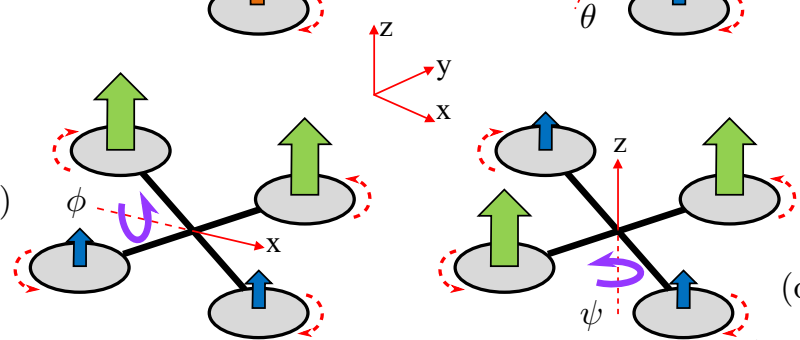

Figura 1. Esquema de movimentação de um quadricóptero na configuração cruzada: (a) thrust, (b) pitch, (c) roll e (d) yaw (adaptada de (Fum, 2015)).

As expressões (1)-(6) são então obtidas por meio da análise dinâmica e cinemática do quadricóptero, supondo corpo rígido, conforme (Raffo, 2011).

$$
\begin{aligned}
\ddot{X} & =-\frac{a_{x}}{m} \dot{X}+\frac{1}{m}(\mathrm{c}(\psi) \mathrm{s}(\theta) \mathrm{c}(\phi)+\mathrm{s}(\psi) \mathrm{s}(\phi)) f_{\mathrm{T}} \\
\ddot{Y} & =-\frac{a_{y}}{m} \dot{Y}+\frac{1}{m}(\mathrm{~s}(\psi) \mathrm{s}(\theta) \mathrm{c}(\phi)-\mathrm{c}(\psi) \mathrm{s}(\phi)) f_{\mathrm{T}} \\
\ddot{z} & =-\frac{a_{z}}{m} \dot{z}-\mathrm{g}+\frac{1}{m} \mathrm{c}(\theta) \mathrm{c}(\phi) f_{\mathrm{T}} \\
\dot{p} & =\left(\frac{J_{y y}-J_{z z}}{J_{x x}}\right) q r+\frac{1}{J_{x x}} \tau_{\phi} \\
\dot{q} & =\left(\frac{J_{z z}-J_{x x}}{J_{y y}}\right) r p+\frac{1}{J_{y y}} \tau_{\theta} \\
\dot{r} & =\left(\frac{J_{x x}-J_{y y}}{J_{z z}}\right) p q+\frac{1}{J_{z z}} \tau_{\psi}
\end{aligned}
$$

onde $f_{\mathrm{T}}$ é o empuxo total gerado pelos rotores, $\tau_{\phi}, \tau_{\theta}, \tau_{\psi}$ os torques produzidos nas respectivas direções angulares $\phi, \theta, \psi, J_{x x}, J_{y y}$ e $J_{z z}$ os momentos de inércia em torno do respectivo eixo, $a_{x}, a_{y}$ e $a_{z}$ são os coeficientes de força de arrasto na respectiva coordenada, $g$ a aceleração da gravidade, e $m$ a massa.

As coordenadas $p, q$ e $r$ são as velocidades angulares no frame do corpo, para obter as velocidades de pitch, roll e yaw é necessária a matriz de rotação em (7).

$$
\left[\begin{array}{c}
\dot{\phi} \\
\dot{\theta} \\
\dot{\psi}
\end{array}\right]=\left[\begin{array}{ccc}
1 & \mathrm{~s}(\psi) \mathrm{s}(\theta) \mathrm{c}(\theta)^{-1} & \mathrm{c}(\psi) \mathrm{s}(\theta) \mathrm{c}(\theta)^{-1} \\
0 & \mathrm{c}(\phi) & -\mathrm{s}(\phi) \\
0 & \mathrm{~s}(\phi) \mathrm{c}(\theta)^{-1} & \mathrm{c}(\phi) \mathrm{c}(\theta)^{-1}
\end{array}\right]\left[\begin{array}{l}
p \\
q \\
r
\end{array}\right]
$$

Para a expressão das acelerações angulares no frame de inércia, $(\ddot{\phi}, \ddot{\theta}, \ddot{\psi})$, basta derivar (7) em relação ao tempo, 
e substituir os respectivos termos do frame do corpo pelos de inércia.

A força e os torques angulares são obtidos por

$$
\boldsymbol{\tau}=\left[\begin{array}{c}
f_{\mathrm{T}} \\
\tau_{\phi} \\
\tau_{\theta} \\
\tau_{\psi}
\end{array}\right]=\left[\begin{array}{cccc}
k_{T} & k_{T} & k_{T} & k_{T} \\
-k_{T} l_{x} & k_{T} l_{x} & k_{T} l_{x} & -k_{T} l_{x} \\
-k_{T} l_{y} & k_{T} l_{y} & -k_{T} l_{y} & k_{T} l_{y} \\
k_{D} & k_{D} & -k_{D} & -k_{D}
\end{array}\right]\left[\begin{array}{c}
\omega_{1}^{2} \\
\omega_{2}^{2} \\
\omega_{3}^{2} \\
\omega_{4}^{2}
\end{array}\right] .
$$

onde $\omega_{i}$ é a velocidade angular do $i$-ésimo rotor, $l_{x}$ e $l_{y}$ são as distâncias entre os rotores e os eixos $X$ e $Y$ do frame do corpo, respectivamente. $k_{T}$ é o coeficiente de empuxo e $k_{D}$ o coeficiente de arrasto das hélices.

\subsection{Representação por Variáveis de Estado}

Como sistema apresentado é naturalmente sub-atuado, pois há apenas 4 variáveis $\left(w_{1}, w_{2}, w_{3}\right.$ e $\left.w_{4}\right)$ para um total de 6 graus de liberdade, não é possível fazer um único controlador atuar em todas os movimentos do quadrirotor. Por esse motivo, é comum em aplicações comerciais o projeto de duas malhas de controle. A primeira para estabilização em referências $z_{r}, \phi_{r}, \theta_{r}$ e $\psi_{r}$ dadas, que terá como saída diretamente o empuxo e o torque dos rotores, e consequentemente a velocidade em cada um. E outra externa que, com base em uma posição ou trajetória de referência no plano $X Y$, determina as referências do segundo controlador.

O objetivo desse trabalho é desenvolver somente o primeiro controlador, para estabilizar os ângulos e a altura do quadrirotor. Portanto, como (3)-(6) não dependem de $X, \dot{X}, Y, \dot{Y}$, as Equações (1)-(2) podem ser ignoradas.

Neste trabalho será suposto que os ângulos serão sempre suficientemente pequenos de modo que podemos aproximar $\mathrm{c}(\cdot) \approx 1$ e $\mathrm{s}(\cdot) \approx 0$. Isso resulta que a matriz de transformação em (7) é aproximada por uma matriz identidade. Além disso, pode ser realizada uma translação $u_{z}=f_{\mathrm{T}}-m g$ para termos o sinal de controle nulo na origem. Assim, é obtido o espaço de estados na forma

$$
\begin{aligned}
& \dot{x}(t)=A(x) x(t)+B(x) u(t), \\
& y(t)=C x(t)+D u(t),
\end{aligned}
$$

onde $x \in \mathbb{R}^{n}, u \in \mathbb{R}^{n_{u}}$ e $A(x), B(x), C, D$ são matrizes de estrutura. Para o quadricóptero considerado, tem-se

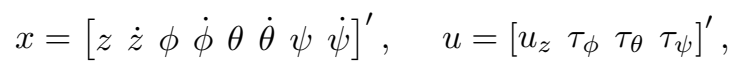

em que $u_{z}=f_{\mathrm{T}}-m \cdot g$,

$$
\begin{aligned}
& A(x)=\left[\begin{array}{cccccccc}
0 & 1 & 0 & 0 & 0 & 0 & 0 & 0 \\
0 & -a_{z} / m & 0 & 0 & 0 & 0 & 0 & 0 \\
0 & 0 & 0 & 1 & 0 & 0 & 0 & 0 \\
0 & 0 & 0 & 0 & 0 & a_{1} x_{8} & 0 & 0 \\
0 & 0 & 0 & 0 & 0 & 1 & 0 & 0 \\
0 & 0 & 0 & a_{2} x_{8} & 0 & 0 & 0 & 0 \\
0 & 0 & 0 & 0 & 0 & 0 & 0 & 1 \\
0 & 0 & 0 & 0 & 0 & 0 & 0 & 0
\end{array}\right],
\end{aligned}
$$

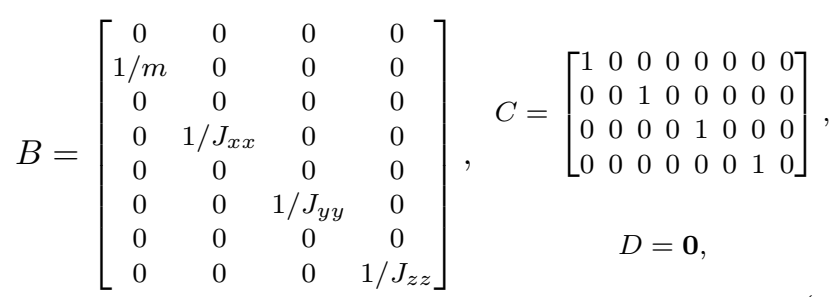

onde $a_{1}=\left(J_{y y}-J_{z z}\right) / J_{x x}$ e $a_{2}=\left(J_{z z}-J_{x x}\right) / J_{y y}$. Note que a matriz $A(x)$ não será linearizada, pois ao contrário da suposição de pequenos ângulos para linearização dos senos e cossenos, não se pode assumir que o produto das velocidades possa ser desprezado, afinal estas podem apresentar excursão considerável.

O sistema é então discretizado utilizando o método ZeroOrder Holder ( $\mathrm{ZOH})$, que assume que as entradas de controle são constantes durante cada período de amostragem $t_{s}$. O sistema em espaços de estados discretos é representado por

$$
\begin{aligned}
x(k+1) & =A_{d} x(k)+B_{d} u(k), \\
y(k) & =C_{d} x(k)+D_{d} u(k),
\end{aligned}
$$

\subsection{Seguimento de Referências Não Nulas}

Para o requisito de erro nulo em regime permanente para entradas do tipo degrau, optou-se pela adição de integradores para eliminar o erro em relação a uma referência $r(k)$ dada. Supôs-se que a função de transferência do integrador discreto $^{1}$ seja (Boyd et al., 1994)

$$
\frac{\xi(z)}{e(z)}=\frac{t_{s}}{z-1},
$$

onde $e(z)=r(z)-y(z)$. A Equação (14) pode ser escrita na forma recursiva, resultando nas Equações (15)-(16).

$$
\begin{aligned}
& x(k+1)=A_{d} x(k)+B_{d} u(k), \\
& \xi(k+1)=\xi(k)+t_{s}(r(k)-y(k)) .
\end{aligned}
$$

Em que a lei de controle associada será da forma

$$
u(k)=K_{e} x(k)+K_{i} \xi(k)=\underbrace{\left[\begin{array}{ll}
K_{e} K_{i}
\end{array}\right]}_{K}\left[\begin{array}{c}
x(k) \\
\xi(k)
\end{array}\right] .
$$

Pode-se assim obter uma representação de estados do sistema com integradores:

$$
\begin{gathered}
\underbrace{\left[\begin{array}{c}
x(k+1) \\
\xi(k+1)
\end{array}\right]}_{x_{a}(k+1)}=\underbrace{\left[\begin{array}{cc}
A_{d} & \mathbf{0} \\
-t_{s} C_{d} & \mathbf{I}
\end{array}\right]}_{A_{a}} \underbrace{\left[\begin{array}{l}
x(k) \\
\xi(k)
\end{array}\right]}_{x_{a}(k)}+\underbrace{\left[\begin{array}{c}
B_{d} \\
\mathbf{0}
\end{array}\right]}_{B_{a}} u(k)+\underbrace{\left[\begin{array}{c}
\mathbf{0} \\
t_{s} \mathbf{I}
\end{array}\right]}_{E_{a}} r, \\
y(k)=\underbrace{\left[\begin{array}{ll}
C_{d} \mathbf{0}
\end{array}\right]}_{C_{a}}\left[\begin{array}{c}
x(k) \\
\xi(k)
\end{array}\right] .
\end{gathered}
$$

\section{REPRESENTAÇÃO POR FRAÇÕES LINEARES}

Para o sistema ser representado por frações lineares, adiciona-se dois sinais auxiliares. Basicamente divide-se o sistema entre dois blocos, um com o sistema linearizado e outro com os estados que causam as não linearidades. Os blocos são interligados pelos sinais auxiliares do sistema, sendo representados por:

$$
\begin{aligned}
\dot{x}(t) & =A x(t)+B u(t)+B_{p} p \\
q & =C_{q} x(t)+D_{q u} u(t)+D_{q p} p \\
p & =\Delta(x) q,
\end{aligned}
$$

onde $x \in \mathbb{R}^{n}$ e $\Delta(x)$ é uma matriz diagonal contendo os elementos de $x$. As matrizes $B_{p}, C_{q}, D_{q u}$ e $D_{q p}$ são determinadas de forma que satisfaça a equação

$$
A(x)=A+B_{p} \Delta(x)\left(\mathbf{I}-D_{q p} \Delta(x)\right)^{-1} C_{q},
$$

1 Neste documento é usada a aproximação por retângulos como método numérico de integração discreta. 
que pode ser obtida a partir de (20).

A discretização é feita de acordo com (Toth et al., 2012) e então adicionados os estados do erro de seguimento de referência, obtendo o sistema LFR discreto aumentado a seguir.

$$
\begin{aligned}
x_{a}(k+1) & =A_{a} x_{a}(k)+B_{a} u(k)+B_{p a} p \\
q & =C_{q a} x_{a}(k)+D_{q u} u(k)+D_{q p} p \\
p & =\Delta(x) q,
\end{aligned}
$$

onde as matrizes $B_{p a}$ e $C_{q a}$ foram aumentadas com zeros, pois os estados do erro não dependem das não linearidades:

$$
B_{p a}=\left[\begin{array}{c}
t_{s} B_{p} \\
\mathbf{0}
\end{array}\right] \quad C_{q a}=\left[\begin{array}{ll}
C_{q} & \mathbf{0}
\end{array}\right] .
$$

Nota-se também que $B_{p a}$ contém o termo multiplicado por $t_{s}$, devido à discretização.

\section{PROJETO DO CONTROLADOR}

Esta seção apresenta os resultados principais do projeto de controladores LFR de tempo discreto concentrados no teorema a seguir.

Teorema 1. Considerando o sistema (22), se existem as matrizes simétricas positivas $Q$ e $T$, a matriz anti-simétrica $H$ e a matriz $L$ de dimensões apropriadas e o escalar positivo $\sigma$ que solucionam o problema LMI a seguir.

$$
\begin{aligned}
& e_{i}^{\prime} Q e_{i}<\sigma^{-2}, \quad i=1, \ldots, n \\
& {\left[\begin{array}{ccc}
Q & \star & \star \\
C_{q a} Q+D_{q u} L & \left(\begin{array}{c}
\sigma^{2} T-D_{q p} H+ \\
H D_{q p}^{\prime}-D_{q p} T D_{q p}^{\prime}
\end{array}\right) & \star \\
A_{a} Q+B_{a} L & -B_{p a} H-B_{p a} T D_{q p}^{\prime} & Q-B_{p a} T B_{p a}^{\prime}
\end{array}\right]>0}
\end{aligned}
$$

então o ganho $K=L Q^{-1}$ estabiliza o sistema e garante que $\mathcal{E} \subset \mathcal{B}$, onde $\mathcal{E}:=\left\{x_{a}: x_{a}^{\prime} P x_{a} \leq 1\right\}$ é um elipsoide que representa uma região de atração do sistema, $\mathcal{B}:=$ $\left\{x:\left|x_{i}\right| \leq \sigma^{-1}, \quad i=1, \ldots, n\right\}$ é uma região definida pela variável $\sigma$ e a lei de controle do sistema é $u(k)=K x_{a}(k)$.

Prova: A prova está estruturada da seguinte maneira: primeiro determina-se uma condição LMI para a positividade e decaimento de da função de Lyapunov discreta $V(k)=x_{a}(k)^{\prime} P x_{a}(k)$ incluindo as não linearidades através de limitação de norma; em seguida desenvolve-se uma condição LMI adicional que garante, através de um elipsoide invariante, que a limitação de norma da não linearidade não será violada pelo projeto. Considerando que $\operatorname{det}((\mathbf{I}-$ $\left.\left.D_{q p} \Delta(x)\right)^{-1}\right) \neq 0, \forall x \in \mathbb{R}^{n}$, e que a matriz $\Delta(x)$ é limitada em norma por

$$
\|\Delta(x)\| \leq \sigma^{-1}
$$

onde $\sigma$ é um escalar positivo, pode-se aplicar o Escalonamento D-G, encontrando que existem as matrizes $S$ e $G$ tais que

$$
\begin{cases}\sigma^{2} p^{\prime} S p \leq q^{\prime} S q, & \forall S=S^{\prime}>0 \\ p^{\prime} G q+q^{\prime} G^{\prime} p=0, & \forall G=-G^{\prime} .\end{cases}
$$

Portanto a restrição em norma pode ser incluída na condição de estabilidade de Lyapunov utilizando o Procedimento$\mathrm{S}$, encontrando

$$
\Delta V\left(x_{a}(k)\right)+p^{\prime} G q+q^{\prime} G^{\prime} p+q^{\prime} S q-p^{\prime} \sigma^{2} S p<0 .
$$

Utilizando a lei de controle $u(k)=K x_{a}(k)$, temos que

$$
\begin{aligned}
A_{f} & =A_{a}+B_{a} K \quad \mathrm{e} \\
C_{f q} & =C_{q a}+D_{q u} K .
\end{aligned}
$$

Assim, após algumas manipulações algébricas, é possível reescrever (28) em forma matricial como

$$
\begin{gathered}
{\left[\begin{array}{cc}
-P & \mathbf{0} \\
\mathbf{0} & -\sigma^{2} S
\end{array}\right]+\left[\begin{array}{c}
A_{f}^{\prime} P \\
B_{p a}^{\prime} P
\end{array}\right] P^{-1}\left[\begin{array}{ll}
P A_{f} & P B_{p a}
\end{array}\right]-\left[\begin{array}{cc}
\mathbf{0} & \mathbf{0} \\
\mathbf{0} & G^{\prime} S^{-1} G
\end{array}\right]} \\
+\left[\begin{array}{c}
C_{f q}^{\prime} \\
G^{\prime} S^{-1}+D_{q p}^{\prime}
\end{array}\right] S\left[\begin{array}{ll}
C_{f q} & S^{-1} G+D_{q p}
\end{array}\right]<0
\end{gathered}
$$

Pode-se inverter os sinais das matrizes, invertendo também o sentido da inequação, adequando assim para utilizar o complemento de Shur. Portanto, tem-se

$$
\begin{gathered}
{\left[\begin{array}{cc}
P & \mathbf{0} \\
\mathbf{0} & \sigma^{2} S
\end{array}\right]-\left[\begin{array}{c}
A_{f}^{\prime} P \\
B_{p a}^{\prime} P
\end{array}\right] P^{-1}\left[\begin{array}{ll}
P A_{f} & P B_{p a}
\end{array}\right]+\left[\begin{array}{cc}
\mathbf{0} & \mathbf{0} \\
\mathbf{0} & G^{\prime} S^{-1} G
\end{array}\right]} \\
-\left[\begin{array}{c}
C_{f q}^{\prime} \\
G^{\prime} S^{-1}+D_{q p}^{\prime}
\end{array}\right] S\left[\begin{array}{ll}
C_{f q} & S^{-1} G+D_{q p}
\end{array}\right]>0
\end{gathered}
$$

Aplicando o complemento de Schur tem-se

$$
\left[\begin{array}{cccc}
P & \star & \star & \star \\
\mathbf{0} & \sigma^{2} S+G^{\prime} S^{-1} G & \star & \star \\
C_{f q} & S^{-1} G+D_{q p} & S^{-1} & \star \\
P A_{f} & P B_{p a} & \mathbf{0} & P
\end{array}\right]>0
$$

Utiliza-se um artifício de multiplicar a matriz pela esquerda e pela direita por diag $\{Q, \mathbf{I}, \mathbf{I}, Q\}$, onde $Q=P^{-1}$, o que resulta em

$$
\left[\begin{array}{cccc}
Q & \star & \star & \star \\
\mathbf{0} & \sigma^{2} S+G^{\prime} S^{-1} G & \star & \star \\
C_{f q} Q & S^{-1} G+D_{q p} & S^{-1} & \star \\
A_{f} Q & B_{p a} & \mathbf{0} & Q
\end{array}\right]>0
$$

Aplicando novamente o complemento de Schur tem-se que

$$
\begin{gathered}
-\left[\begin{array}{c}
\mathbf{0} \\
S^{-1} G+D_{q p} \\
B_{p a}
\end{array}\right]\left(\sigma^{2} S+G^{\prime} S^{-1} G\right)^{-1}\left[\begin{array}{c}
\mathbf{0} \\
S^{-1} G+D_{q p} \\
B_{p a}
\end{array}\right]^{\prime} \\
+\left[\begin{array}{ccc}
Q & Q C_{f q}^{\prime} & Q A_{f}^{\prime} \\
C_{f q} Q & S^{-1} & \mathbf{0} \\
A_{f} Q & \mathbf{0} & Q
\end{array}\right]>0
\end{gathered}
$$

A partir das mudanças de variáveis $T=\left(\sigma^{2} S+\right.$ $\left.G^{\prime} S^{-1} G\right)^{-1}$ e $H=T\left(S^{-1} G\right)^{\prime}$ encontra-se

$$
\begin{array}{r}
-\left[\begin{array}{ccc}
\mathbf{0} & \mathbf{0} & \mathbf{0} \\
\mathbf{0} & \left(\begin{array}{c}
S^{-1} G\left(\sigma^{2} S+G^{\prime} S^{-1} G\right)^{-1} G^{\prime} S^{-1} \\
+D_{q p} H+H^{\prime} D_{q p}^{\prime}+D_{q p} T D_{q p}^{\prime}
\end{array}\right) & \left(\begin{array}{c}
H^{\prime} B_{p a}^{\prime}+ \\
D_{q p} T B_{p a}^{\prime}
\end{array}\right) \\
\mathbf{0} & B_{p a} H+B_{p a} T D_{q p}^{\prime} & \\
B_{p a} T B_{p a}^{\prime}
\end{array}\right] \\
+\left[\begin{array}{ccc}
Q & Q C_{f q}^{\prime} & Q A_{f}^{\prime} \\
C_{f q} Q & S^{-1} & \mathbf{0} \\
A_{f} Q & \mathbf{0} & Q
\end{array}\right]>0
\end{array}
$$

Um dos termos da matriz pode ser simplificado sabendo-se que (Petersen and Pedersen, 2012)

$$
S^{-1}-S^{-1} G\left(\sigma^{2} S+G^{\prime} S^{-1} G\right)^{-1} G^{\prime} S^{-1}=\sigma^{2} T .
$$

Portanto, temos que

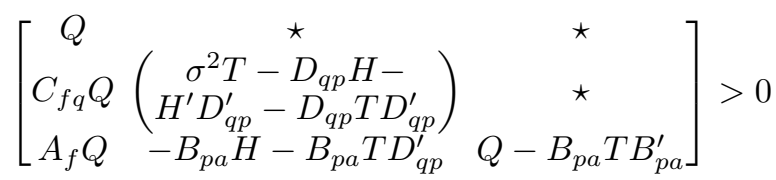

e com $A_{f}$ e $C_{q f}$ de (29) e definindo $L=K Q$, tem-se

$$
\left[\begin{array}{cc}
Q & \star \\
C_{q a} Q+D_{q u} L & \left.\begin{array}{c}
\sigma^{2} T-D_{q p} H+ \\
H D_{q p}^{\prime}-D_{q p} T D_{q p}^{\prime}
\end{array}\right) \\
A_{a} Q+B_{a} L & -B_{p a} H-B_{p a} T D_{q p}^{\prime} Q-B_{p a} T B_{p a}^{\prime}
\end{array}\right]>0 .
$$


Considere um elipsoide invariante definido como $\mathcal{E}:=$ $\left\{x_{a}: x_{a}^{\prime} P x_{a} \leq 1\right\}$ e a região $\mathcal{B}:=\left\{x:\left|x_{i}\right| \leq \sigma^{-1}\right.$, $i=1, \ldots, n\}$. De acordo com (El Ghaoui and Scorletti, 1996), se o elipsoide $\mathcal{E}$ é uma região de atração do sistema (22), então a LMI

$$
\left[\begin{array}{cc}
\sigma^{-2} & e_{i}^{\prime} \\
e_{i} & P
\end{array}\right]>0,
$$

onde $e_{i}$ é a $i$-ésima coluna de uma matriz identidade de dimensão $n+n_{y}$, garante que $\mathcal{E}$ está contido em $\mathcal{B}$. Aplicando o complemento de Schur e $Q=P^{-1}$ tem-se

$$
\sigma^{-2}-e_{i}^{\prime} Q e_{i}>0
$$

Não é desejável que o controlador seja agressivo ao ponto de levar os ângulos a serem grandes, inviabilizando a aproximação das funções seno e cosseno do modelo. Para evitar isso, considere o corolário a seguir.

Corolário 2. Suponha que exista solução para as LMIs do Teorema 1 acrescidas da condição LMI a seguir.

$$
\left[\begin{array}{ll}
U & L \\
L^{\prime} & Q
\end{array}\right] \geq 0, \quad U:=\operatorname{diag}\left\{\bar{u}_{1}^{2}, \ldots, \bar{u}_{n_{u}}^{2}\right\} .
$$

onde $\bar{u}_{i}, i \in \mathbb{R}^{n_{u}}$, representa o valor máximo desejado para a a ação de controle $u_{i}$. Então, as propriedades resultantes do Teorema 1 são mantidas e o controle é limitado.

Prova: A prova completa pode ser encontrada em (Kothare et al., 1996). Resumidamente, a condição (41) impõe que a lei de controle não ultrapassa os limites preestabelecidos para todo $x_{a} \in \mathcal{E}$.

\section{RESULTADOS}

Ao analisar as equações de $\dot{p}, \dot{q}$ e $\dot{r}$ em (1)-(6), observase a existência do produto de duas velocidades angulares em cada um desses termos. Diferente dos ângulos que podem ser assumidos pequenos e desprezados, os produtos $q r, p r$ e $p q$ foram considerados como perturbações na representação LFR. Assim, tem-se as seguintes matrizes:

$$
\begin{aligned}
& \Delta(x)=\left[\begin{array}{cc}
x_{8} & 0 \\
0 & x_{8}
\end{array}\right], \quad B_{p}=\left[\begin{array}{cccccccc}
0 & 0 & 0 & 0 & 0 & a_{2} & 0 & 0 \\
0 & 0 & 0 & a_{1} & 0 & 0 & 0 & 0
\end{array}\right]^{\prime}, \\
& C_{q}=\left[\begin{array}{lllllllll}
0 & 0 & 0 & 1 & 0 & 0 & 0 & 0 \\
0 & 0 & 0 & 0 & 0 & 1 & 0 & 0
\end{array}\right], \quad D_{q u}=\mathbf{0}, \quad D_{q p}=\mathbf{0},
\end{aligned}
$$

e considerou-se $\sigma=1$ como limite desejado para este caso.

O quadricóptero Iris da empresa 3DR (3DR, 2018), é utilizado para validação por ser um modelo comercial e possuir modelo no simulador Gazebo. Os parâmetros do quadricóptero determinados em (Fum, 2015) são apresentados na Tabela 1.

Os valores máximos desejados para as ações de controle são apresentados na Tabela 2, sendo calculados a partir da análise das equações do modelo, adotando $\bar{u}_{z}$ de $2 m g$ e a variação máxima $\Delta w$ que cada torque pode alterar da velocidade final dos rotores. As LMIs foram resolvidas utilizado o solver SeDuMi e o parser YALMIP (Löfberg, 2004) no software Matlab.

O Robot Operation System (ROS) é um framework flexível para o desenvolvimento de software para robótica (ROS, 2019). Na coleção de ferramentas e bibliotecas disponíveis,
Tabela 1. Parâmetros Iris 3DR.

\begin{tabular}{ccc}
\hline Parâmetro & Valor & Unidade \\
\hline \hline$g$ & 9,81 & $\mathrm{~m} / \mathrm{s}^{2}$ \\
$m$ & 1,37 & $\mathrm{~kg}$ \\
$l_{x}$ & 0,13 & $\mathrm{~m}$ \\
$l_{y}$ & 0,21 & $\mathrm{~m}$ \\
$k_{T}$ & $15,67 \cdot 10^{-6}$ & $\mathrm{~N} \cdot \mathrm{s}^{2} / \mathrm{rad}^{2}$ \\
$k_{D}$ & $2,55 \cdot 10^{-7}$ & $\mathrm{~N} \cdot \mathrm{m} \cdot \mathrm{s}^{2} / \mathrm{rad}^{2}$ \\
$J_{x x}$ & 0,0219 & $\mathrm{~kg} \cdot \mathrm{m}^{2}$ \\
$J_{y y}$ & 0,0109 & $\mathrm{~kg} \cdot \mathrm{m}^{2}$ \\
$J_{z z}$ & 0,0306 & $\mathrm{~kg} \cdot \mathrm{m}^{2}$ \\
$a_{x}$ & 0,25 & $\mathrm{~kg} / \mathrm{s}$ \\
$a_{y}$ & 0,25 & $\mathrm{~kg} / \mathrm{s}$ \\
$a_{z}$ & 0,25 & $\mathrm{~kg} / \mathrm{s}$ \\
\hline
\end{tabular}

Tabela 2. Valores máximos da ação de controle.

\begin{tabular}{ccc}
\hline Parâmetro & Valor & Unidade \\
\hline \hline $\bar{u}_{z}$ & 26,88 & $\mathrm{~N}$ \\
$\bar{\tau}_{\phi}$ & 0,60 & $\mathrm{~N} \cdot \mathrm{m}$ \\
$\bar{\tau}_{\theta}$ & 0,30 & $\mathrm{~N} \cdot \mathrm{m}$ \\
$\bar{\tau}_{\psi}$ & 0,10 & $\mathrm{~N} \cdot \mathrm{m}$ \\
\hline
\end{tabular}

está o Gazebo, simulador desenvolvido para o teste de algoritmos, projeto de robôs, treino de inteligências artificias em sistemas indoor e outdoor (Gazebo, 2019).

Assim, o conjunto modelo e controlador é validado por meio de simulações do modelo não linear (1)-(6), e com o simulador Gazebo utilizando o framework ROS. O plugin desenvolvido está disponível para acesso público ${ }^{2}$.

Nas simulações é utilizada uma sequência de set-points de referência, alterados à cada 10 segundos. Apenas um estado é alterado por vez, com exceção da última mudança, que altera simultaneamente todas as referências. Os resultados obtidos no Gazebo são apresentados nas Figuras 2-5.

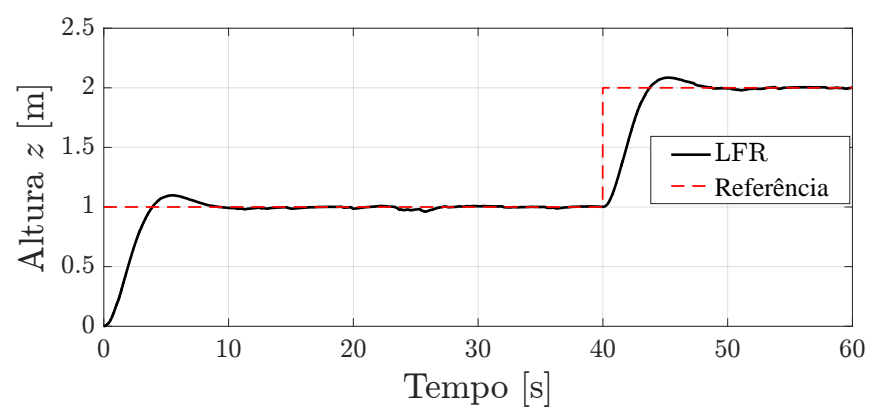

Figura 2. Resultados altura $z$ no Gazebo.

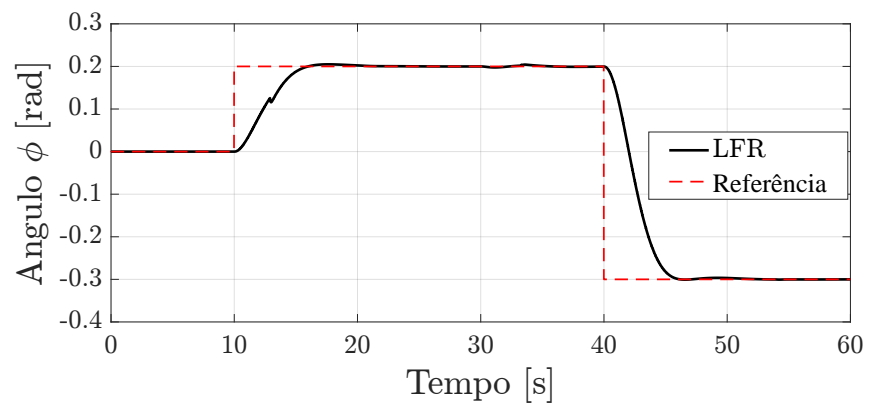

Figura 3. Ângulo $\phi$ simulado no Gazebo.

O comportamento dos torques é ilustrado na Figura 7, onde nota-se eventuais perturbações em instantes aleató-

\footnotetext{
2 https://github.com/Schulze18/iris_plugin_robust_control.
} 


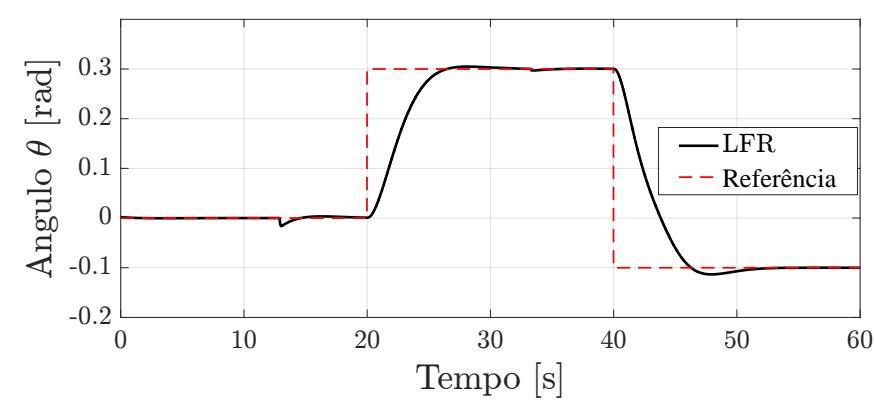

Figura 4. Ângulo $\theta$ simulado no Gazebo.

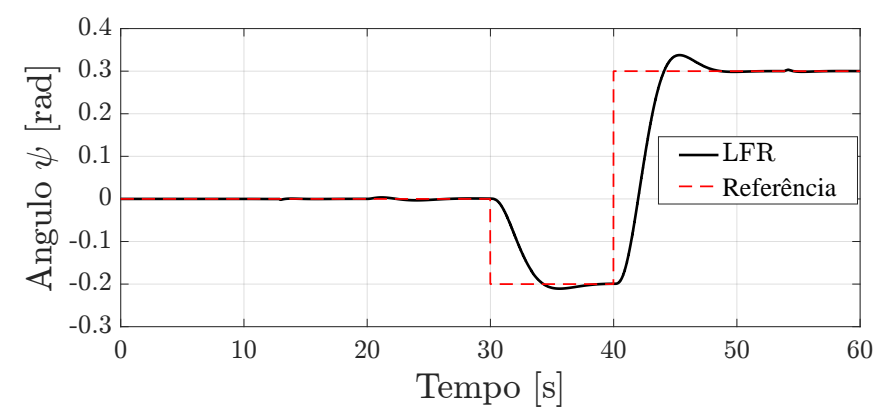

Figura 5. Ângulo $\psi$ simulado no Gazebo.

rios no Gazebo. Tais picos e descontinuidades são inerentes à forma como o Gazebo realiza a solução da otimização numérica de sistemas físicos (Okoli et al., 2019) e variam a cada simulação. As mesmas simulações apresentadas nesta seção foram realizadas utilizando o Matlab/Simulink com o modelo matemático do sistema (omitidas aqui por restrições de espaço), resultando em curvas coincidentes com as das simulações do Gazebo, exceto pelos instantes de pico nos sinais de controle. As simulações evidenciam que os limites de controle foram atendidos.

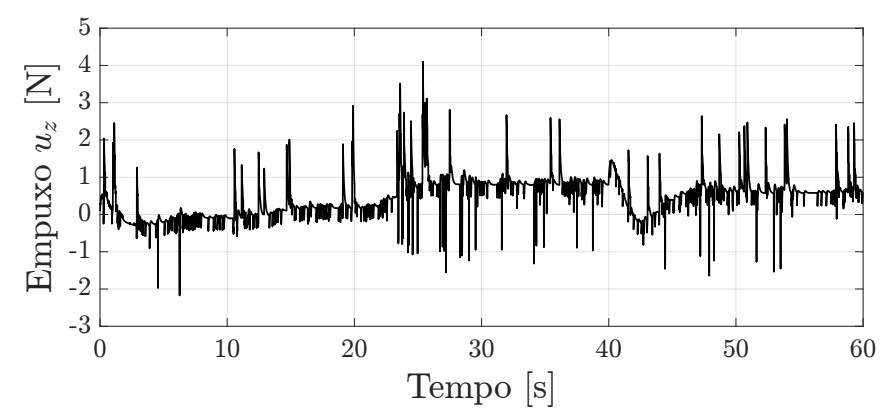

Figura 6. Empuxo $u_{z}$ simulado no Gazebo.

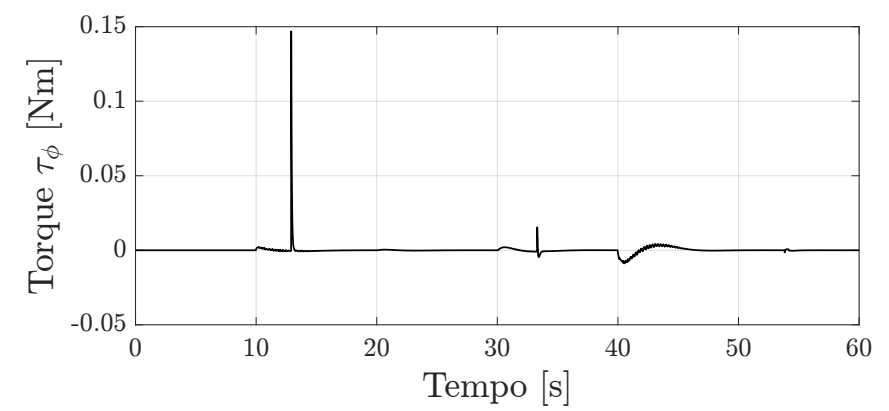

Figura 7. Torque $\tau_{\phi}$ simulado no Gazebo.

\section{CONCLUSÕES}

Este artigo apresentou o desenvolvimento de condições LMI para o projeto de controladores para sistemas não lineares com representação do tipo LFR de tempo discreto. A técnica foi estendida para a imposição de limitações no sinal de controle e sua aplicação foi ilustrada em um sistema quadrirotor através de simulações numéricas. Tais simulações foram realizadas utilizando o framework ROS/Gazebo para validação do modelo matemático em malha fechada. Possíveis extensões da técnica de controle incluem o tratamento de incertezas paramétricas e observadores de estados. Do ponto de vista do sistema quadrirotor tem-se o desafio da extensão para tratar as não linearidades senoidais e cossenoidais de ângulos grandes e a validação experimental.

\section{REFERÊNCIAS}

3DR (2018). Iris+ | 3DR. Disponível em: https://3dr. com/support/articles/iris/, Acesso em 19/03/2019. Boyd, S., El Ghaoui, L., Feron, E., and Balakrishnan, V. (1994). Linear Matrix Inequalities in System and Control Theory, volume 86. SIAM, Philadelphia, USA.

El Ghaoui, L. and Scorletti, G. (1996). Control of rational systems using linear-fractional representations and linear matrix inequalities. Automatica, 32(9).

Fum, W.Z. (2015). Implementation of Simulink controller design on Iris + quadrotor. Master's thesis, Naval Postgraduate School, Monterey, California.

Gazebo (2019). Gazebo - robot simulation made easy. Disponível em: http://gazebosim.org/, Acesso em 27/03/2019.

Grüne, L. (1997). Discrete feedback stabilization of nonlinear control systems at a singular point. In 1997 European Control Conference (ECC), 3200-3203.

Kothare, M.V., Balakrishnan, V., and Morari, M. (1996). Robust constrained model predictive control using linear matrix inequalities. Automatica, 32(10), 1361 - 1379.

Löfberg, J. (2004). Yalmip : a toolbox for modeling and optimization in matlab. In 2004 IEEE International Conference on Robotics and Automation (IEEE Cat. No.04CH37508).

Okoli, F., Lang, Y., Kermorgant, O., and Caro, S. (2019). Cable-driven parallel robot simulation using gazebo and ros. CISM International Centre for Mechanical Sciences, Courses and Lectures, 288-295.

Petersen, K.B. and Pedersen, M.S. (2012). The matrix cookbook.

Pozo, F., Ikhouane, F., and Rodellar, J. (2008). Numerical issues in backstepping control: Sensitivity and parameter tuning. Journal of the Franklin Institute, 345(8), 891 -905 .

Raffo, G.V. (2011). Robust Control Strategies for a QuadRotor Helicopter - An Underactuated Mechanical System. Ph.D. thesis, Universidad de Sevilla, Sevilla.

ROS (2019). ROS.org | Powering the world's robots. Disponível em: http://www.ros.org/, Acesso em $27 / 03 / 2019$.

Toth, R., Lovera, M., Heuberger, P.S.C., Corno, M., and den Hof, P.M.J.V. (2012). On the discretization of linear fractional representations of lpv systems. IEEE Transactions on Control Systems Technology, 20(6). 